\title{
Upregulation of miR-216a exerts neuroprotective effects against ischemic injury through negatively regulating JAK2/STAT3-involved apoptosis and inflammatory pathways
}

\author{
Yu Shuang Tian, MD, Di Zhong, PhD, Qing Qing Liu, MD, Xiu Li Zhao, PhD, Hong Xue Sun, MD, \\ Jing Jin, MD, Hai Ning Wang, MD, and Guo Zhong Li, PhD
}

Department of Neurology, The First Affiliated Hospital, Harbin Medical University, Harbin, Heilong Jiang Province, China

\begin{abstract}
OBJECTIVE Ischemic stroke remains a significant cause of death and disability in industrialized nations. Janus tyrosine kinase (JAK) and signal transducer and activator of transcription (STAT) of the JAK2/STAT3 pathway play important roles in the downstream signal pathway regulation of ischemic stroke-related inflammatory neuronal damage. Recently, microRNAs (miRNAs) have emerged as major regulators in cerebral ischemic injury; therefore, the authors aimed to investigate the underlying molecular mechanism between miRNAs and ischemic stroke, which may provide potential therapeutic targets for ischemic stroke.

METHODS The JAK2- and JAK3-related miRNA (miR-135, miR-216a, and miR-433) expression levels were detected by real-time quantitative reverse-transcriptase polymerase chain reaction (qRT-PCR) and Western blot analysis in both oxygen-glucose deprivation (OGD)-treated primary cultured neuronal cells and mouse brain with middle cerebral artery occlusion (MCAO)-induced ischemic stroke. The miR-135, miR-216a, and miR-433 were determined by bioinformatics analysis that may target JAK2, and miR-216a was further confirmed by $3^{\prime}$ untranslated region (3'UTR) dual-luciferase assay. The study further detected cell apoptosis, the level of lactate dehydrogenase, and inflammatory mediators (inducible nitric oxide synthase [iNOS], matrix metalloproteinase-9 [MMP-9], tumor necrosis factor- $\alpha$ [TNF- $\alpha$ ], and interleukin$1 \beta[\mathrm{IL}-1 \beta]$ ) after cells were transfected with miR-NC (miRNA negative control) or miR-216a mimics and subjected to oxygen-glucose deprivation/reoxygenation (OGD/R) damage with 3-(4,5-dimethylthiazol-2-yl)-2,5-diphenyltetrazolium bromide (MTT) assay, annexin V-FITC/PI, Western blots, and enzyme-linked immunosorbent assay detection. Furthermore, neurological deficit detection and neurological behavior grading were performed to determine the infarction area and neurological deficits.
\end{abstract}

RESULTS JAK2 showed its highest level while miR-216a showed its lowest level at day 1 after ischemic reperfusion. However, miR-135 and miR-433 had no obvious change during the process. The luciferase assay data further confirmed that miR-216a can directly target the 3'UTR of JAK2, and overexpression of miR-216a repressed JAK2 protein levels in OGD/R-treated neuronal cells as well as in the MCAO model ischemic region. In addition, overexpression of miR-216a mitigated cell apoptosis both in vitro and in vivo, which was consistent with the effect of knockdown of JAK2. Furthermore, the study found that miR-216a obviously inhibited the inflammatory mediators after OGD/R, including inflammatory enzymes (iNOS and MMP-9) and cytokines (TNF- $\alpha$ and IL-1 $\beta$ ). Upregulating miR-216a levels reduced ischemic infarction and improved neurological deficit.

CONCLUSIONS These findings suggest that upregulation of miR-216a, which targets JAK2, could induce neuroprotection against ischemic injury in vitro and in vivo, which provides a potential therapeutic target for ischemic stroke.

https://thejns.org/doi/abs/10.3171/2017.5.JNS163165

KEY WORDS miR-216a; JAK2/STAT3; MCAO; ischemic injury

ABBREVIATIONS CCA = common carotid artery; DMEM = Dulbecco's modified Eagle's medium; ELISA = enzyme-linked immunosorbent assay; FBS = fetal bovine serum; GAPDH = glyceraldehyde 3-phosphate dehydrogenase; ICA = internal carotid artery; IL-1 = interleukin-1; iNOS = inducible nitric oxide synthase; JAK = Janus tyrosine kinase; $\mathrm{LDH}=$ lactate dehydrogenase; $\mathrm{MCAO}=$ middle cerebral artery occlusion; miRNA = microRNA; miR-NC = miRNA negative control; MMP-9 = matrix metalloproteinase-9; MTT = 3-(4,5-dimethylthiazol-2-yl)-2,5-diphenyltetrazolium bromide; OGD = oxygen-glucose deprivation; OGD/R = oxygen-glucose deprivation/reoxygenation; OGD2/ $\mathrm{R} 24$ = 2 hours of OGD and 24 hours of reoxygenation; PCN = primary cortical neuronal; qRT-PCR = quantitative reverse-transcriptase polymerase chain reaction; SDS-

PAGE = sodium dodecyl sulfate-polyacrylamide gel electrophoresis; siJAK2 = JAK2 short-interfering RNA; STAT = signal transducer and activator of transcription; TNF- $\alpha=$ tumor necrosis factor- $\alpha$; TTC = 2,3,5-triphenyltetrazolium chloride; TUNEL = terminal deoxynucleotidyl transferase-mediated deoxyuridine triphosphate nick-end labeling; $3^{\prime} \mathrm{UTR}=3^{\prime}$ untranslated region.

SUBMITTED December 19, 2016. ACCEPTED May 8, 2017.

INCLUDE WHEN CITING Published online March 9, 2018; DOI: 10.3171/2017.5.JNS163165. 
I SCHEMIC stroke is the second most common cause of death and disability in industrialized nations, accounting for $11 \%$ of the total deaths in $2013 .{ }^{8}$ Transient focal cerebral ischemia has long been known to be related to increased proinflammatory cytokines, including tumor necrosis factor- $\alpha$ (TNF- $\alpha$ ), interleukin-1 (IL-1), and IL6 , finally leading to postischemic inflammatory neuronal damage, including cell apoptosis, cerebral edema, and other cellular reactions, such as angiogenesis. ${ }^{6,11,24}$ Janus tyrosine kinase (JAK) and signal transducer and activator of transcription (STAT) play important roles in the downstream signal pathway regulation of these cytokines. The JAK/STAT pathway is reported to be involved in the cellular response to various extracellular stimuli, such as ischemic stress..$^{10,15}$ To date, there is no sufficient therapeutic approach for this obstinate illness; thus, it is of paramount importance to find an effective approach to protect the brain against ischemic injury. ${ }^{4}$

MicroRNAs (miRNAs), commonly known as endogenous single-stranded noncoding RNA molecules, have been discovered to negatively modulate protein expression by degradation or repression of their target mRNAs via sequence complementarity. ${ }^{2}$ These findings add a new level of posttranscriptional regulatory control for the expression of genes. Researchers have identified differentially expressed miRNAs in stroke patients and mouse or rat middle cerebral artery occlusion (MCAO) models using large-scale miRNA microarrays. ${ }^{5,12}$ More importantly, miRNAs have been found to be involved in the pathophysiology of ischemic stroke, which can result in altered neurotransmitter function, oxidative metabolism, protein chaperones, and apoptotic processes. , $^{3,30}$

The study of miR-216a has mainly been focused on its diagnostic value in tumors. For example, miR-216a was found to be significantly repressed in esophageal squamous cell carcinoma ${ }^{7}$ and human pancreatic cancer ${ }^{27,31}$ and was upregulated in hepatocellular carcinoma development via regulation of its target genes. ${ }^{29}$ However, the study of miR-216a in ischemic injury was limited and the exact role of miR-216a in this type of injury was unclear. In this study, we aimed to identify the potential relationship between miR-216a and JAK2 and investigate its role in JAK2/STAT3-mediated ischemic injury. To be able to elucidate this mechanism will provide us new information in the treatment of ischemic stroke.

\section{Methods \\ Isolation of Primary Cortical Neuronal Cells}

Neonatal Sprague-Dawley mice were used for the isolation of primary cortical neuronal (PCN) cells. Briefly, the cerebral cortices were digested $(0.25 \%$ trypsin at $37^{\circ} \mathrm{C}$ ) for 1 hour and then incubated in wells precoated with $0.1 \mathrm{mg} / \mathrm{ml}$ poly-L-lysine. The cells were cultured in Dulbecco's modified Eagle's medium (DMEM) containing $10 \%$ fetal bovine serum (FBS) and $10 \mu \mathrm{mol} / \mathrm{L}$ cytosine- $\beta$-D-arabinofuranoside (catalog no. C1768, Sigma). Three hours later, the culture solution was replaced by Neurobasal-A medium (catalog no. 10888022, Thermo Fisher Scientific) containing 2\% B27 NeuroMix, $10 \mu \mathrm{M}$ cytosine- $\beta$-D-arabinofuranoside, $100 \mathrm{U} / \mathrm{ml}$ penicillin, and $100 \mathrm{U} / \mathrm{ml}$ streptomycin.

\section{Oxygen-Glucose Deprivation/Reoxygenation Model}

The isolated PCN cells were cultured in glucose-free Earle's balanced salt solution and immediately transferred to a humidified anaerobic chamber containing an atmosphere of $1 \% \mathrm{O}_{2}, 94 \% \mathrm{~N}_{2}$, and $5 \% \mathrm{CO}_{2}$ for 2 hours. When the oxygen-glucose deprivation/reoxygenation (OGD/R) model was fixed, the culture solution was replaced with normal medium and cultured for 3 hours, 12 hours, 1 day, 2 days, 3 days, and 7 days. The control group was not deprived of oxygen and glucose and was incubated in standardized DMEM (containing normal oxygen and glucose). For the miRNA experiment, PCN cells were pretreated with miR-216a mimics or miRNA negative control (miR-NC) for 24 hours.

\section{Cell Transfection}

PCN cells were first plated in 96-well or 6-well plates, followed by mixing with lipofectamine 2000 (Invitrogen) according to the manufacturer's instructions. When cells were $70 \%$ confluent, they were transfected with miR-216a mimics (100 nM, GenePharma), miR-NC (50 nM, GenePharma), and JAK2 short-interfering RNA (siJAK2; 50 $\mathrm{nM}$, GenePharma) at the indicated time.

\section{Cell Apoptosis, Lactate Dehydrogenase Detection, and Inflammatory Mediator Assays}

PCN cells were transfected with miR-NC or miR-216a mimics and then subjected to OGD/R damage (the cells were exposed to 2 hours of OGD and then 3 hours, 12 hours, 1 day, 2 days, 3 days, and 7 days of reoxygenation). We used the 3-(4,5-dimethylthiazol-2-yl)-2,5-diphenyltetrazolium bromide (MTT) assay kit and annexin V-FITC/ PI analysis to detect cell apoptosis according to the manufacturer's instructions. The detection of intracellular lactate dehydrogenase (LDH) and inflammatory mediators was performed by lyses with $0.2 \%$ Triton X-100 for 15 minutes and measured at $490 \mathrm{~nm}$ by a spectrophotometer. In addition, the level of IL- $1 \beta$ and TNF- $\alpha$ in medium was measured using enzyme-linked immunosorbent assay (ELISA) kits.

\section{Real-Time Polymerase Chain Reaction for miRNAs}

Total RNA was isolated from the cortical peri-infarct area of the ipsilateral hemisphere or PCN cells using the Trizol method. The separated and purified RNA was then reverse transcribed into cDNA using the Revert Aid First Strand cDNA Synthesis Kit (Thermo Fisher Scientific) on the Bio-Rad CFX96 Detection System, followed by real-time quantitative reverse-transcriptase polymerase chain reaction (qRT-PCR) on the Plexor One-Step qRTPCR System (Promega). The primers for miRNA amplification were as follows: rno-mir-135-S: 5'-TGCGGTATG GCTTTTTATTCC-3'; rno-mir-135-AS: 5'-CCAGTGCAG GGTCCGAGGT-3'; rno-mir-216a-S: 5'-TGCGGTAAT CTCAGCTTGGCA-3'; rno-mir-216a-AS: 5'-CCAGTG CAGGGTCCGAGGT-3'; rno-mir-433-S: 5'-TGCGGA TCATGATGGGCTCCT-3'; rno-mir-433-AS: 5'-CCA GTGCAGGGTCCGAGGT-3'; U6-S: 5' TGCGGGTGC TCGCTTCGGCAGC-3'; and U6-AS: 5'-CCAGTGCAG GGTCCGAGGT-3'. 
The fold change in relative miRNA expression was determined using the $2^{-\Delta \Delta C t}$ method, as described previously. ${ }^{22}$

\section{Luciferase Reporter Assay}

The wild type of the $3^{\prime}$ untranslated region ( $3^{\prime}$ UTR) of the JAK2 gene (including miR-216a binding sites) and mutant 3'UTR of the JAK2 gene were synthesized by RiboBio and cloned into the downstream portion of the pmirGLO vector (Promega) to generate JAK2-3'UTR and mutant JAK2-3'UTR (JAK2-3'mUTR), which were confirmed by sequencing. For the luciferase reporter assay, PCN cells were seeded in 24-well plates at a density of $2 \times 10^{4}$ cells per well. When the cells reached $70 \%$ confluency, they were cotransfected with either JAK2-3'UTR (100 ng) or JAK2-3'mUTR (100 ng) and miR-216a or miR-216a-mut mimics $(100 \mathrm{nM})$ or miR-NC mimics $(50 \mathrm{nM})$. Forty-eight hours later, cells were harvested and assayed using the Dual-Luciferase Reporter Assay System (Promega) according to the manufacturer's instruction. Each experiment was independently repeated more than 3 times. The primers used were as follows: 1) JAK2-3'UTR-wt-Top: SAL1 5'-CTAGCTAGCGGCCGCTAGTTGTTATTGGTGTCT TCACTGGATG-3'; 2) JAK2-3'UTR-wt-Bot: NHEL 5'-TCGACATCCAGTGAAGACACCAATAACAAC TAGCGGCCGCTAG-3'; 3) JAK2-3'UTR-mt-Top: SAL1 5'-CTAGCTAGCGGCCGCTAGTTGTTATTGGTGTCT TCACTGGATG-3'; and 4) JAK2-mt-3'UTR-Bot: NHEL 5'-TCGACATCCAGTGAAGACACCAATAACAACTAG CGGCCGCTAG-3'.

\section{Western Blot Analysis and ELISA}

The isolated total protein was separated on $10 \%$ sodium dodecyl sulfate-polyacrylamide gel electrophoresis (SDSPAGE) and then transferred to polyvinylidene difluoride membranes (GE Healthcare), followed by blocking with $10 \%$ skimmed milk for 1 hour at room temperature. The membranes were then incubated with the primary antibodies: JAK2 (1:1000, Santa Cruz Biotechnology, Inc.), p-STAT3 (1:1000, Santa Cruz Biotechnology), inducible nitric oxide synthase (iNOS; 1:1000, Cell Signaling, Inc.), matrix metalloproteinase-9 (MMP-9; 1:1000, Chemicon International, Inc.), or cleaved caspase-3 (1:1000, Cell Signaling). The secondary antibody was a horseradish peroxidase-conjugated secondary antibody, goat antirabbit (Stressgen Biotechnologies). After incubation of the membranes with the secondary antibody, the signal bands were visualized by chemiluminescence (GE Healthcare). The quantification was performed using the GelDoc-2000 Imagine System (Bio-Rad). Glyceraldehyde 3-phosphate dehydrogenase (GAPDH) was used as an internal control to normalize the protein levels. We also used the PathScan Phospho-Stat3 Sandwich ELISA Kit (Cell Signaling) to explore the expression of p-STAT3 in the cells undergoing different treatments (normal, OGD/R, miR-216a, pcDNA3-JAK2, or miR-216a+pcDNA3-JAK2) according to a previous study. ${ }^{28}$

\section{Animals and Surgery}

This study was approved and supervised by the Insti- tutional Animal Care and Use Committee of the First Affiliated Hospital of Harbin Medical University. Male mice were housed in individual cages with free access to food and water (12-hour light/dark cycles at $22^{\circ} \pm 2{ }^{\circ} \mathrm{C}$ ). The MCAO model of ischemic stroke was conducted using the following steps. Mice were deeply anesthetized with an intraperitoneal injection of pentobarbital sodium (40 $\mathrm{mg} / \mathrm{kg}$ ). A blunt dissection was performed under a stereomicroscope (Stemi 2000, Carl Zeiss) to expose the left common carotid artery (CCA), followed by ligation of the ipsilateral CCA proximal end and external carotid artery and clamping of the internal carotid artery (ICA) with an arterial clamp. This was followed by a small incision in the CCA between permanent and temporary sutures, in which a 5-0 surgical nylon filament with a round tip $(0.23$ $\mathrm{mm}$ in diameter) was inserted into the ICA approximately $12 \mathrm{~mm}$ beyond the carotid bifurcation, thereby occluding the origin of the middle cerebral artery. After 2 hours of $\mathrm{MCAO}$, the mice were allowed to recover for 3 hours, 12 hours, 1 day, 2 days, 3 days, and 7 days ( 3 mice in each group, 18 mice total). The mice in the sham group $(n=3)$ were subjected to the same procedure except for the insertion of an intraluminal filament. We used laser Doppler flowmetry (PeriFlux System 5000, Perimed) to monitor the regional cerebral blood flow at a location $0.5 \mathrm{~mm}$ anterior and $5.0 \mathrm{~mm}$ lateral to the bregma. The rectal temperature was monitored using a temperature-controlled heating pad (CMA 150, Carnegie Medicin) during and after surgery. The blood glucose levels were tested with Accu-Chek Performa (Roche).

\section{miRNA Treatment for the MCAO Model}

For miRNA treatment, the mice were anesthetized with chloral hydrate $(10 \%)$ and then placed in the stereotactic apparatus (anteroposterior $0.8 \mathrm{~mm}$, mediolateral $1.5 \mathrm{~mm}$, depth 3.5 mm; series 68001, RWD Life Science Co., Ltd.). After anesthesia, the mice were subjected to the injection of miR-216a mimics (7 $\mu \mathrm{l}$ of $100 \mu \mathrm{M}$, purchased from GenePharma) into the left-brain lateral ventricle for 20 minutes in a total volume of $7 \mu \mathrm{l}, 10$ minutes after MCAO. The injection was considered successful only when cerebral blood flow dropped to the baseline level (before occlusion) and was maintained for at least 20 minutes during injection. To confirm the overexpression of miR-424, cortical tissue was harvested from each cerebral hemisphere $2 \mathrm{~mm}$ around the site of injection and processed for realtime qRT-PCR. After 24 hours of reperfusion, all mice were then used to conduct neurological deficit assessment.

\section{Determination of Infarction Area and Neurological Deficits}

The detection of neurological deficits was performed according to the standard procedure of the Garcia scoring method $^{18,20} 24$ hours after reperfusion. The neurological behavior was graded on a 10-point scale on the basis of the observations. The mice were then killed with a lethal dose of sodium pentobarbital for the determination of infarction area. The mice were transcardially perfused with sodium pentobarbital to remove intravascular blood. The brains of all the mice were removed and sliced into five $1.5-\mathrm{mm}-$ thick coronal sections at the indicated time ( 3 hours, 12 hours, 1 day, 2 days, 3 days, and 7 days after MCAO) with 
mouse brain matrix. The coronal brain sections $(2 \mathrm{~mm}$ thick) were stained with 2,3,5-triphenyltetrazolium chloride (TTC; Sigma). Areas of infarct tissue were measured using Image-Pro Plus software (Media Cybernetics, Inc.). The percentage of infarction was calculated using the following formula: infarct volume/total volume of ischemic cerebral hemisphere $\times 100 \%$.

\section{Statistical Analysis}

All data were expressed as means \pm SDs, and statistical analysis was performed using SPSS (version 11.5, SPSS, Inc.). Differences were assessed by 1-way ANOVA and post hoc Scheffe tests; $p<0.05$ was considered statistically significant.

\section{Results \\ Alteration of JAK2 and miRNA Expression in PCN Cells After OGD/R}

To investigate the role of JAK2 and potential miRNAs that may target JAK2, we performed bioinformatic analysis; the results showed that miR-216a, miR-135, and miR433 may target JAK2 (Fig. 1A). We also detected the expression of miRNAs in the OGD/R model (PCN cells) at different times ( 3 hours, 12 hours, 1 day, 2 days, 3 days, and 7 days). The real-time qRT-PCR results indicated that the expression of miR-216a was downregulated in the OGD/R model compared with the expressions of miR-135 and miR-433 (Fig. 1B-D). In addition, the level of miR216a was at its lowest on day 1 and decreased to its lowest level on that day (Fig. 1B). We also observed a high level of JAK $2 \mathrm{mRNA}$ in the OGD/R model. The levels of JAK2 mRNA elevated gradually in a time-dependent manner and reached the highest level on day 1 , followed by a gradual decrease (Fig. 1E). The Western blot data indicated the level of JAK2 reaching the highest level on day 1, which was similar to the trend of JAK 2 mRNA (Fig. 1F and G).

\section{miR-216a Directly Targets JAK2 in PCN Cells}

To further investigate whether miR-216a directly targets JAK2 mRNA, the wild-type $3^{\prime}$ UTR or mutant $3^{\prime}$ UTR of JAK2 mRNA was ligated to downstream of the firefly luciferase gene in the pmirGLO plasmid (Promega) to generate JAK2 $-3^{\prime}$ UTR and JAK2 $-3^{\prime}$ mUTR plasmids. The JAK2-3'UTR and JAK2-3'mUTR plasmids were cotransfected with miR-NC or miR-216a mimics into PCN cells. As shown in Fig. 2A, the luciferase activity results showed that the luciferase activity of PCN cells cotransfected with miR-216a mimics and JAK2-3'UTR was obviously reduced. However, the luciferase activity of PCN cells cotransfected with miR-216a mimics and JAK2-3'mUTR had no significant change. Furthermore, we performed Western blot analysis and real-time qRTPCR to investigate the role of miR-216a and JAK2 in the $\mathrm{OGD} / \mathrm{R}$ model. The PCN cells were transfected with miR$\mathrm{NC}$ or miR-216a mimics for 24 hours and then subjected to 2 hours of OGD and 24 hours of reoxygenation (OGD2/ R24). The real-time qRT-PCR data showed that the level of JAK2 mRNA was significantly inhibited in the group of cells transfected with miR-216a mimics (Fig. 2B). The Western blot findings suggested that the expression of
JAK2 protein was also decreased in the miR-216a mimics group compared with the miR-NC group and was similar to the expression of JAK2 mRNA (Fig. 2C and D). All these data indicated that miR-216a directly targets JAK2 via binding to the $3^{\prime}$ UTR of JAK2 mRNA.

\section{miR-216a Reduced PCN Cell Apoptosis Mediated by JAK2 in the OGD/R Model}

To investigate the role of miR-216a and JAK2 in the OGD/R model, PCN cells were transfected with miR-216a mimics and siJAK2 for 24 hours and then subjected to OGD2/R24 at the indicated times (24, 48, and 72 hours). The MTT results showed that the viability of PCN cells in the OGD/R group was significantly decreased, and the decrease showed time dependence (Fig. 3A). However, the viability of cells was restored by the introduction of miR-216a mimics or siJAK2 (Fig. 3A). At the same time, the LDH level of the OGD/R group was significantly increased compared with the level of the normal group, while overexpression of miR-216a mimics or knocking down JAK 2 could repair the damage (Fig. 3B). The terminal deoxynucleotidyl transferase-mediated deoxyuridine triphosphate nick-end labeling (TUNEL) results showed that inhibition of JAK2 alleviated OGD2/R24-induced PCN cell death compared with the normal group (Fig. 3C and D). We also explored the expression of p-STAT3 and cleaved caspase- 3 in 3 groups of cells (normal group, OGD/R group, and siJAK2 group). The Western blot data showed that the level of p-STAT3 and cleaved caspase-3 was significantly promoted in the OGD/R group and inhibition of JAK 2 could restore the increased level of pSTAT3 and cleaved caspase-3 induced by OGD2/R24 (Fig. 3E). Consistently, the ELISA results indicated that the expression of p-STAT3 was upregulated in the OGD/R group and inhibition of JAK2 could partly restore the increased level of p-STAT3 (Fig. 3F and G). All these findings suggested that miR-216a-reduced PCN cell apoptosis in the OGD/R model may be mediated by JAK2.

\section{miR-216a Decreased Neuroinflammation and Apoptosis via the JAK2/STAT3 Pathway After OGD/R Damage}

Because activation of the JAK2/STAT3 pathway was reported to be involved in ischemia, ${ }^{13,25}$ we next aimed to explore the role of miR-216a and the JAK2/STAT3 pathway in the OGD/R model. We cotransfected miR-216a, pcDNA3-JAK2, and miR-216a+pcDNA3-JAK2 into PCN cells and cultured them for 24 hours. The TUNEL results showed that OGD2/R24 and overexpression of JAK2 lead to increased cell apoptosis compared with normal cells. Introduction of miR-216a could restore the increased cell apoptosis (induced by OGD2/R24 and pcDNA3-JAK2); however, the protective effect of miR-216a could be weakened by overexpression of JAK2 (miR-216a+pcDNA3JAK2; Fig. 4A and E). From Fig. 4B and F, we can see that the expression of protein p-STAT3, JAK2, and cleaved caspase-3 were strongly increased in the OGD/R group compared with the normal group. In particular, transfection of miR-216a mimics in the OGD/R model significantly decreased the expression level of p-STAT3, JAK2, and cleaved caspase- 3 protein, and the effect can be restored 
A $\mathrm{miR}-433$

\begin{tabular}{|c|c|}
\hline & \\
\hline Rat & - \\
\hline Human & UGCUA-GC-----AUUUU------GAGAUUAAGAA-----UUUAAGCU---UA-A-GCCAUAAAAUAG- \\
\hline Chimp & -GC-----AUUUU------GAGAUUAAGAA-----UUUAAGCU---UA-A-GCCAUAAAA---- \\
\hline Rhesus & GC-----AUGUU------GAGAUUAAGAA---- \\
\hline Squirrel & CUUG--A----UGCCC-AC----UUUUU----GAAAUUAAGAA----UUUAAAUA---UA-A-GCCAUAAAA--- \\
\hline Mouse & ---AUCAUG--A-----UGCUA-GC----AUUUU-----GAGAUUAAGAA----UUGAAGCA---UA-A-GCCAUA-CA \\
\hline Rabbit & JA-GU-----AGUUU------GA \\
\hline & GAA-----UUUAA \\
\hline
\end{tabular}

B

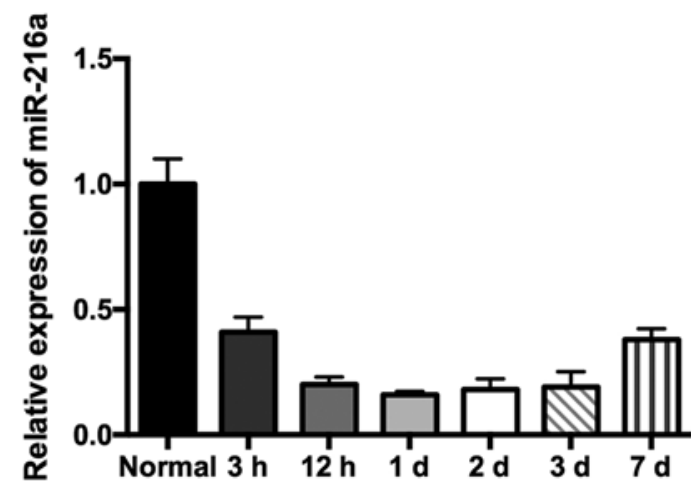

D

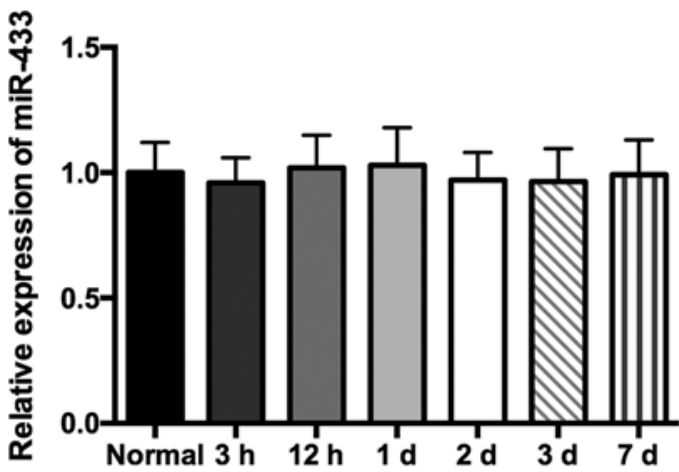

$\mathbf{F}$

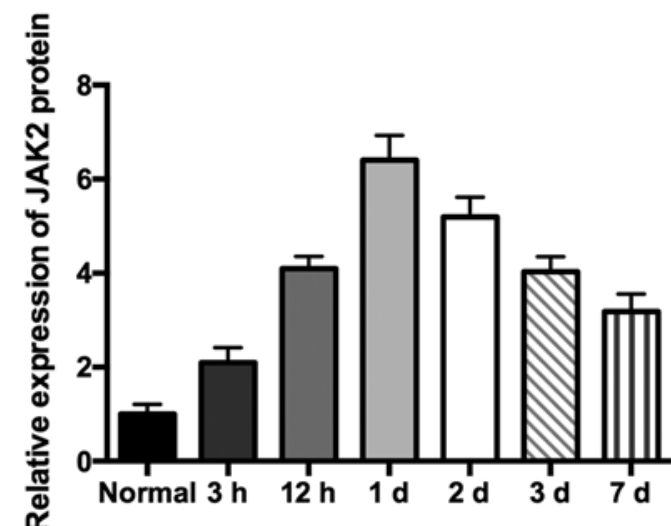

C

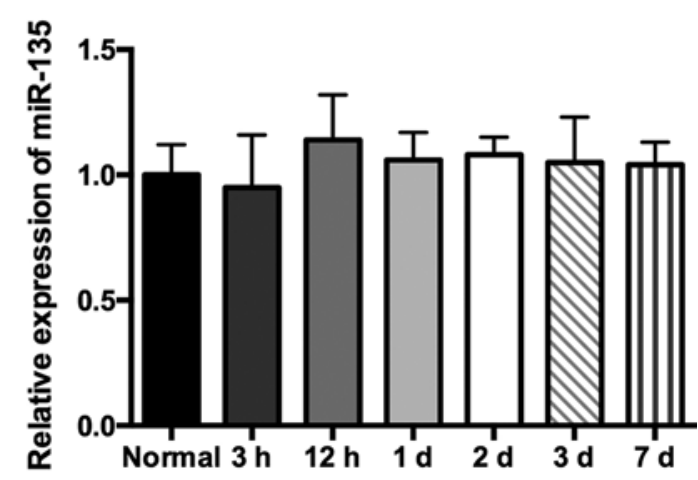

E

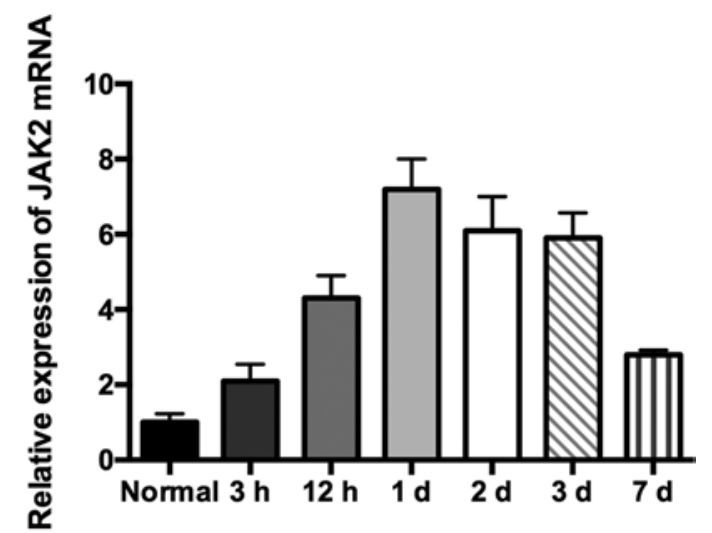

G

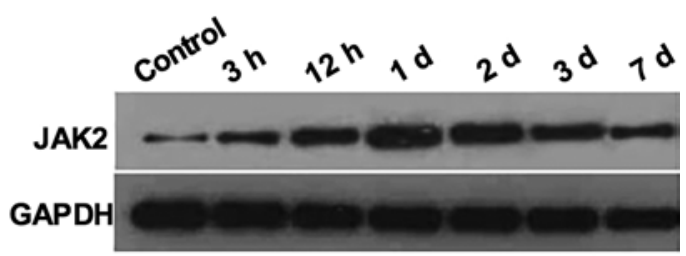

FIG. 1. Changes in JAK2 and miRNA expression in OGD/R-induced neuronal cells. A: Scheme of 3 potential miRNAs that may target JAK2 through different species. B-D: The changes in miR-216a (B), miR-135 (C), and miR-433 (D) expression levels were measured using real-time qRT-PCR. The small nucleolar RNA (SNRNA) U6 was used as an internal control for quantification of the miRNAs. E-G: The expression levels of JAK2 mRNA (E) and protein ( $F$ and $G$ ) were measured. GAPDH was used as an internal control.

by the introduction of pcDNA3-JAK2. The ELISA results showed that the expression of p-STAT3 in the miR-216a group was significantly increased in the pcDNA3-JAK2 group and transfection of miR-216a mimics can inhibit the increased p-STAT3 induced by overexpression of JAK2 (Fig. 4D), which was consistent with the Western blot analysis. We further investigated downstream inflammatory mediators (iNOS, MMP-9, TNF- $\alpha$, and IL-1 $\beta$ ) of the 
A

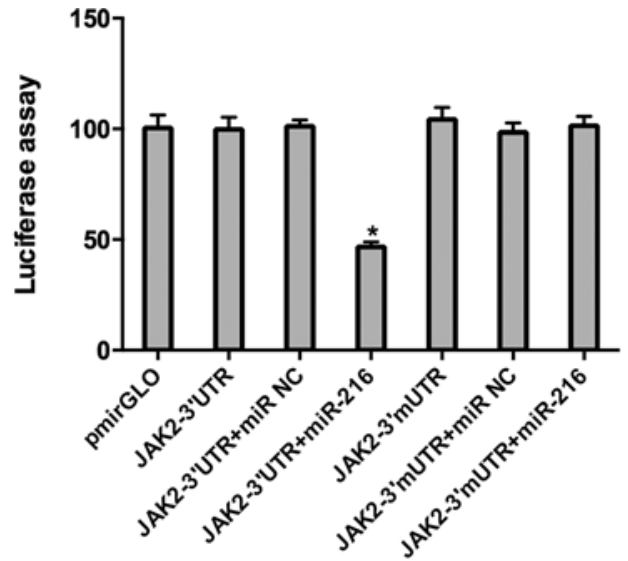

B

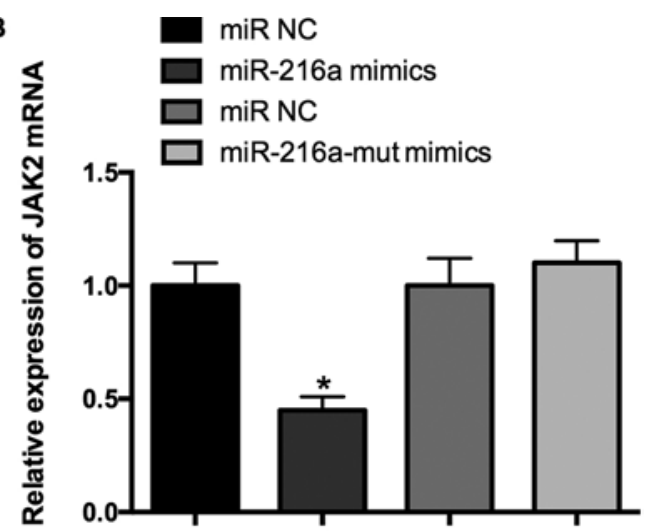

C

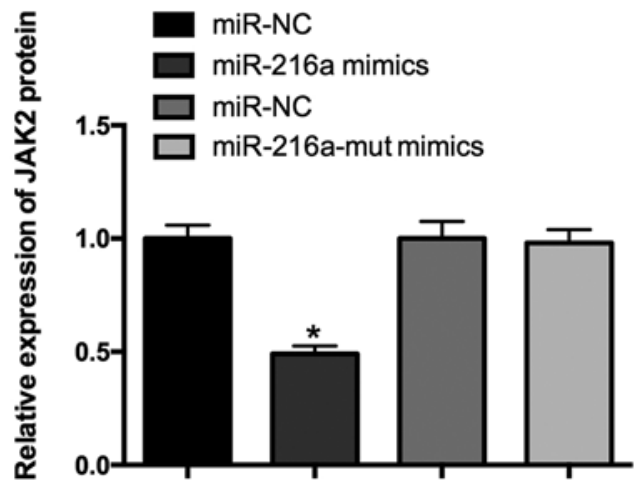

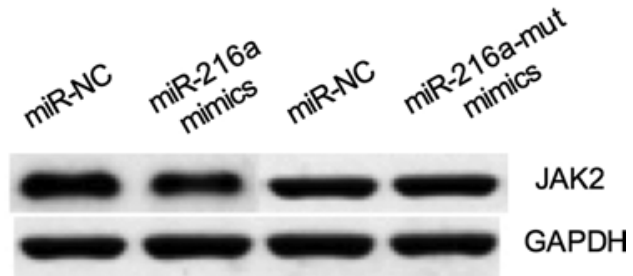

FIG. 2. miR-216a directly recognized the $3^{\prime} U T R$ of JAK2 mRNAs in OGD-treated neuronal cells. A: Luciferase reporter assay results demonstrated that cotransfection of the luciferase reporter containing 3'UTR of JAK2 with miR-216a resulted in a decrease in luciferase activity. B: JAK2 mRNA levels were measured by using real-time qRT-PCR. GAPDH was used as an internal control for quantification of mRNA levels. There was decreased expression of JAK2 mRNA with miR-216a treatment. C: Quantitative analysis showed that miR-216a reduced JAK2 protein levels in neuronal cells. D: Representative results of Western blot analysis show the changes of JAK2 protein levels after being transfected with miR-NC and miR-216a mimics. ${ }^{*} p<0.05$.

JAK2/STAT3 pathway. As shown in Fig. 4C, the levels of IL-1 $\beta$ and TNF- $\alpha$ were strongly increased in the OGD/R group and pcDNA3-JAK2 group compared with normal cells, and the increase could be inhibited by the introduction of miR-216a mimics. However, the protective effect of miR-216a could be reduced by cotransfection with pcDNA3-JAK2. Furthermore, miR-216a mimics reduced the upregulated expression of iNOS and MMP-9 induced by OGD/R and pcDNA3-JAK2. Consistent with TNF- $\alpha$ and IL-1 $\beta$, the effect of miR-216a could be weakened by knockdown of JAK2 (Fig. 4G). All these results indicated that miR-216a clearly decreased neuroinflammation and apoptosis via the JAK2/STAT3 pathway after OGD/R damage.

\section{Downregulation of miR-216a and Correlation With Activation of JAK2 In Vivo}

To further confirm the exact role of miR-216a in cerebral ischemic injury in vivo, we injected miR-216a mimics into the mouse cortex. The expression level of miR216a was confirmed by real-time qRT-PCR (Fig. 5A). All samples were taken from the peri-infarct region of mice after 2 hours of MCAO and reperfusion at the indicated time. As shown in Fig. 5A, the expression level of miR216a was downregulated in the MCAO group compared with the sham group and reached its lowest level on day 1. We also observed a high level of JAK2 immunoreactive cells in ischemic mouse brains, especially on day 1 (highest level; Fig. 5B), which had a similar trend in PCN cells. These data indicated that the expression of miR-216a was correlated with the expression of JAK2 in cerebral ischemic injury.

\section{Reintroduction of miR-216a and Downregulation of the JAK2/STAT3 Pathway In Vivo}

As we noted above, the JAK2/STAT3 pathway can be activated after ischemic injury, and we further detected the expression of p-STAT3 and its downstream mediators (cleaved caspase-3 and iNOS) in vivo. The immunohistochemistry results suggested that the p-STAT3 protein level was increased 3 hours after MCAO and sustained to 7 days, while reaching its peak on day 1 . The introduction of miR-216a significantly inhibited the protein level of pSTAT3 (Fig. 6A). The expression of cleaved caspase-3 and iNOS was also promoted in the MCAO group compared with the sham group, and injection of miR-216a mimics could restore the damage (promoted level of cleaved caspase-3 and iNOS) induced in MCAO (Fig. 6B and C). Considering that the reintroduction of miR-216a led to the inhibited expression of JAK2, we suspected that reintro- 

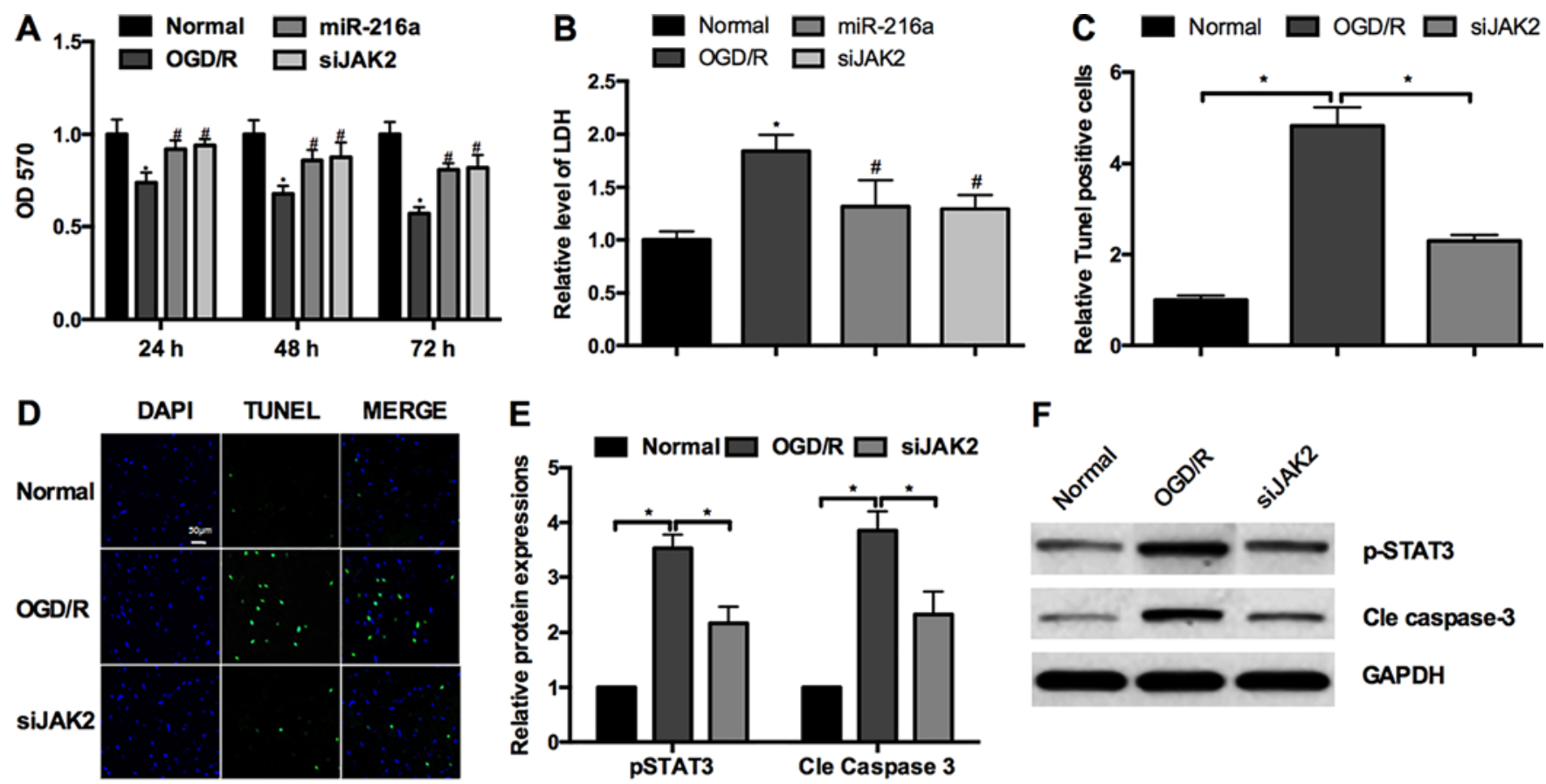

$\mathbf{F}$
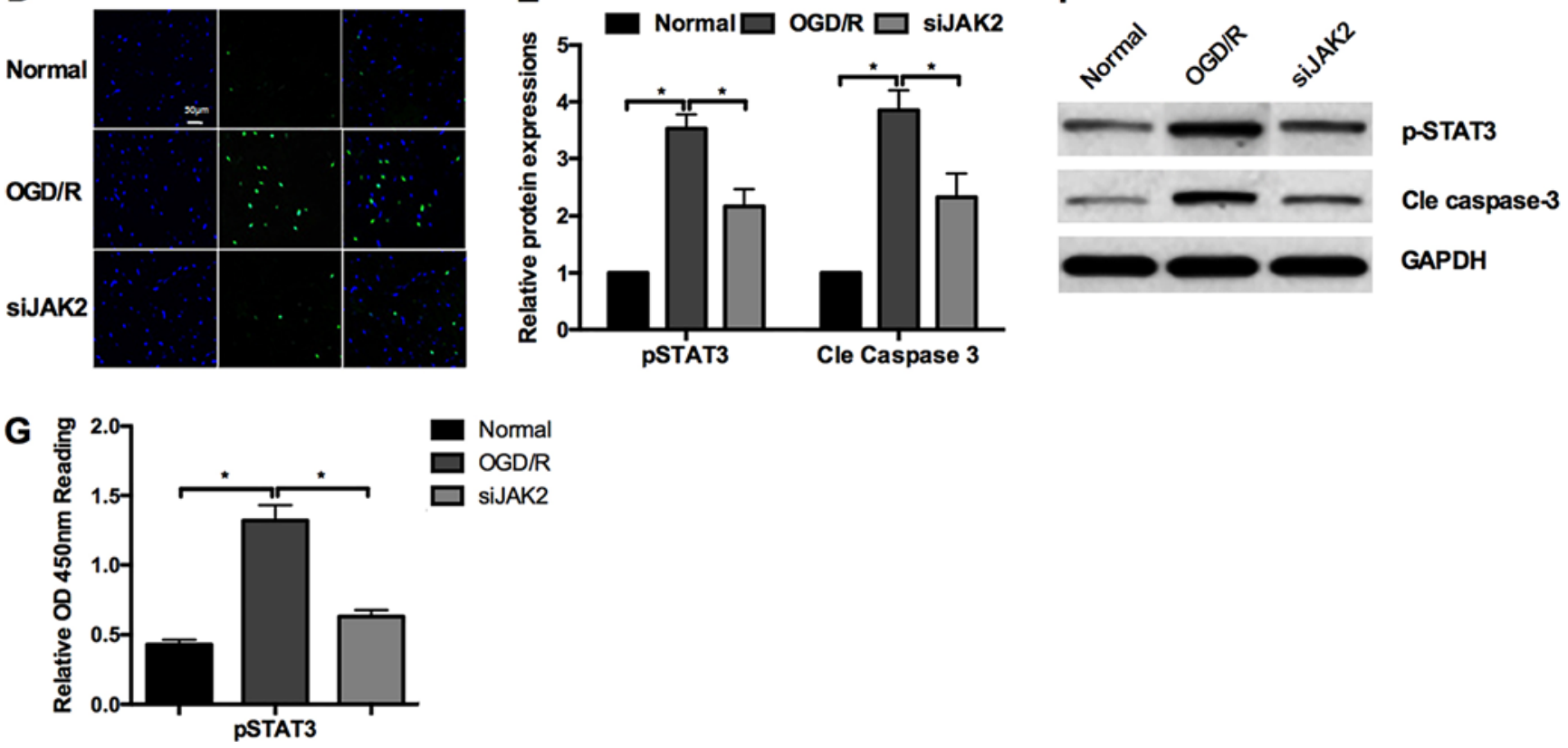

FIG. 3. The effect of miR-216a and siJAK2 on PCN cells. A: Cell viability was detected by MTT assay. B: The LDH level was detected. C: Quantitative analysis showed that knockdown of JAK2 can decrease TUNEL-positive cells in OGD2/R24-treated neuronal cells. D: TUNEL assays. E and F: Quantitative analysis (E) and Western blot (F) showed that knockdown of JAK2 can decrease protein levels of p-STAT3 and cleaved caspase-3 (Cle caspase-3). G: ELISA analysis showed that the expression of p-STAT3 was promoted in the OGD/R group and inhibition of JAK2 partly decreased the upregulated p-STAT3 level induced by OGD/R. ${ }^{*} p<0.05 ; \# p<0.05$ represents a comparison with the OGD/R group. Figure is available in color online only.

duction of miR-216a could attenuate the neuroinflammation in MCAO via downregulation of the JAK2/STAT3 pathway in vivo.

\section{Reintroduction of miR-216a and Decreased Ischemic Infarction, Neuronal Death, and Neurological Dysfunction In Vivo}

We also performed neurological deficit detection to investigate the effect of miR-216a in vivo. The TTC analysis showed that the infarct volume was significantly increased in the ischemia/reperfusion group compared with the sham group and the promoted infarct volume induced in MCAO can be attenuated by injection of miR-216a mimics (Fig. 7A and B). The neurological deficit results showed that miR-216a decreased neurological deficit compared with MCAO models (Fig. 7C and D). The cerebral blood flow of mice in the MCAO group and MCAO+miRNA216a group showed no difference during and after brain ischemia. The blood glucose levels and rectal tempera- ture $\left(37.0^{\circ} \mathrm{C}\right)$ in mice from the MCAO group $(8.53 \pm$ $1.62 \mathrm{mmol} / \mathrm{L})$ and $\mathrm{MCAO}+\mathrm{miR}-424$ group $(8.57 \pm 2.04$ $\mathrm{mmol} / \mathrm{L}$ ) were essentially identical. Consequently, the TUNEL assay confirmed that miR-216a can reduce brain cell apoptosis (Fig. 7E). Collectively, these results indicated that introduction of miR-216a could exert a neuroprotective effect in the ischemic brain by downregulation of the JAK2/STAT3 pathway in vivo.

\section{Discussion}

Neuronal cell death is considered a critical part of stroke pathophysiology, and a variety of miRNAs have been identified that exert their effects in the progression of ischemic injury by inducing cell death of the brain. ${ }^{1}$ For example, miR-124 was reported to reduce neuronal loss in mouse focal cerebral ischemia through regulating the expression of apoptosis-stimulating protein of p53.16 Peng and colleagues found that inhibiting miR-181b induced reduction of ischemic neuronal death by repressing its tar- 
A

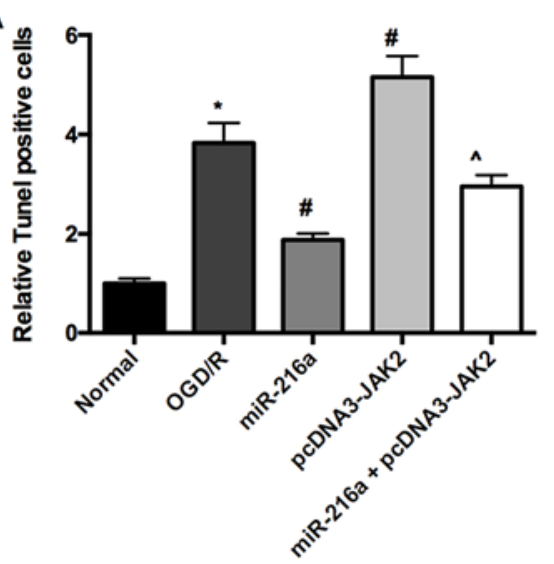

B

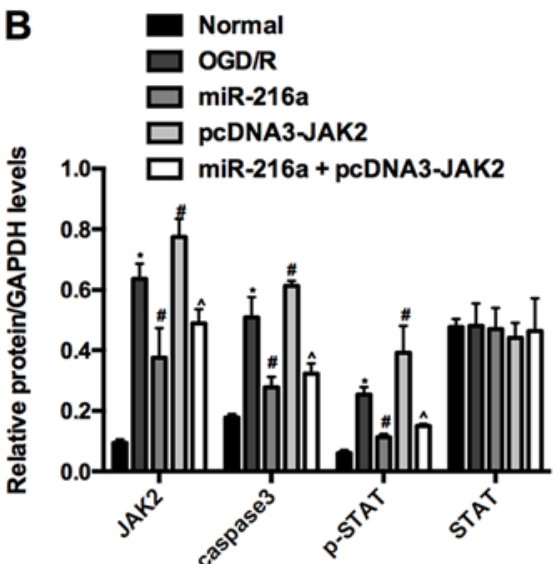

C

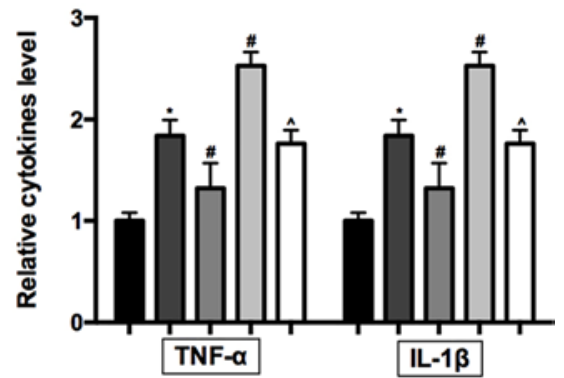

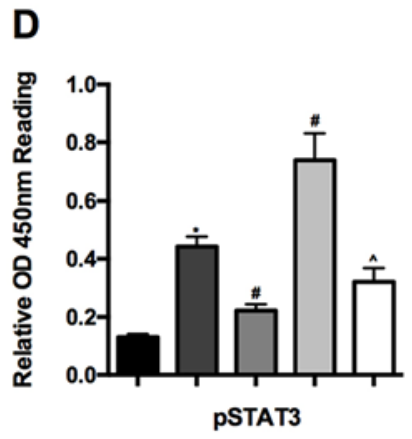

E
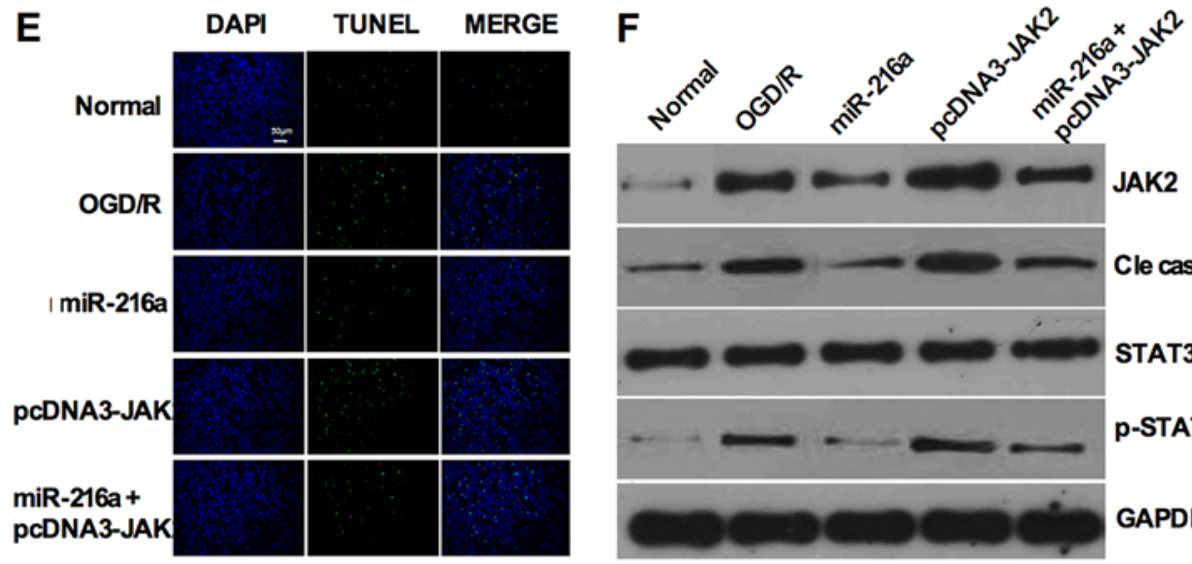

JAK2

Cle caspase-3

STAT3

p-STAT3

GAPDH

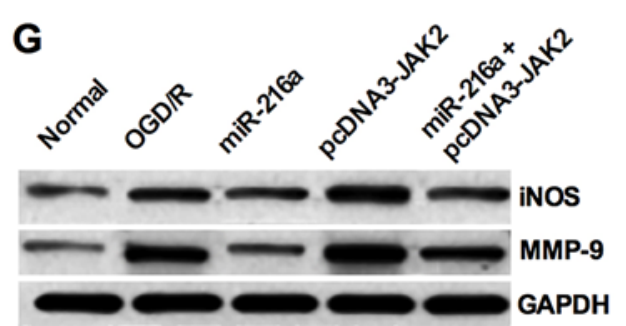

FIG. 4. Overexpression of JAK2 can restore miR-216a-repressed JAK2/STAT3 activation and apoptosis in OGD2/R24-treated PCN cells. A: Quantitative analysis showed that miR-216a can decrease TUNEL-positive cells, while JAK2 can increase TUNELpositive cells. Cotreatment with JAK2 and miR-216a can reverse this trend. B: Quantitative analysis showed that miR-216a can decrease protein levels of JAK2, p-STAT3, and cleaved caspase-3, while JAK2 can increase these protein levels. Cotreatment with miR-216a and JAK2 can reverse this trend. C: The levels of IL-1 $\beta$ and TNF- $\alpha$ were increased in the OGD/R group and pcDNA3-JAK2 group compared with normal cells, and the increase could be inhibited by introduction of miR-216a mimics. D: The ELISA results showed that the expression of p-STAT3 in the miR-216a group was increased in the pcDNA3-JAK2 group and transfection of miR-216a mimics can inhibit the increased p-STAT3 induced by overexpression of JAK2. E: TUNEL assays. F: Western blot was performed. G: miR-216a mimics reduced the upregulated expression of iNOS and MMP-9 induced by OGD/R and pcDNA3-JAK2, and the effect of miR-216a can be weakened by knockdown of JAK2. ${ }^{*} p<0.05 ;{ }^{\wedge} p<0.05$ represents a comparison with the pcDNA3-JAK2 group; $\# p<0.05$ represents a comparison with the OGD/R group. Figure is available in color online only.

get mRNA translation in vitro and in vivo..$^{19}$ In the present study, we first found that the expression of miR-216a was inhibited in OGD/R-treated neuronal cells and the brains of MCAO mice, whereas JAK2 and p-STAT3 were upregulated. In addition, knockdown of JAK2 or overexpression of miR-216a can play neuroprotective roles in vitro and in vivo.

Accumulated evidence has confirmed that the JAK2/
STAT3 pathway can be activated by cerebral ischemia and JAK2/STAT3 plays a key role in the pathological process of stroke. ${ }^{14}$ Downregulation of the JAK2/STAT3 pathway was also correlated with upregulation of miRNAs. For example, miR-101 inhibits proliferation of pulmonary microvascular endothelial cells in a rat model of hepatopulmonary syndrome by targeting the JAK2/STAT3 signaling pathway. ${ }^{26}$ Hou et al. found that miR-216a may function 

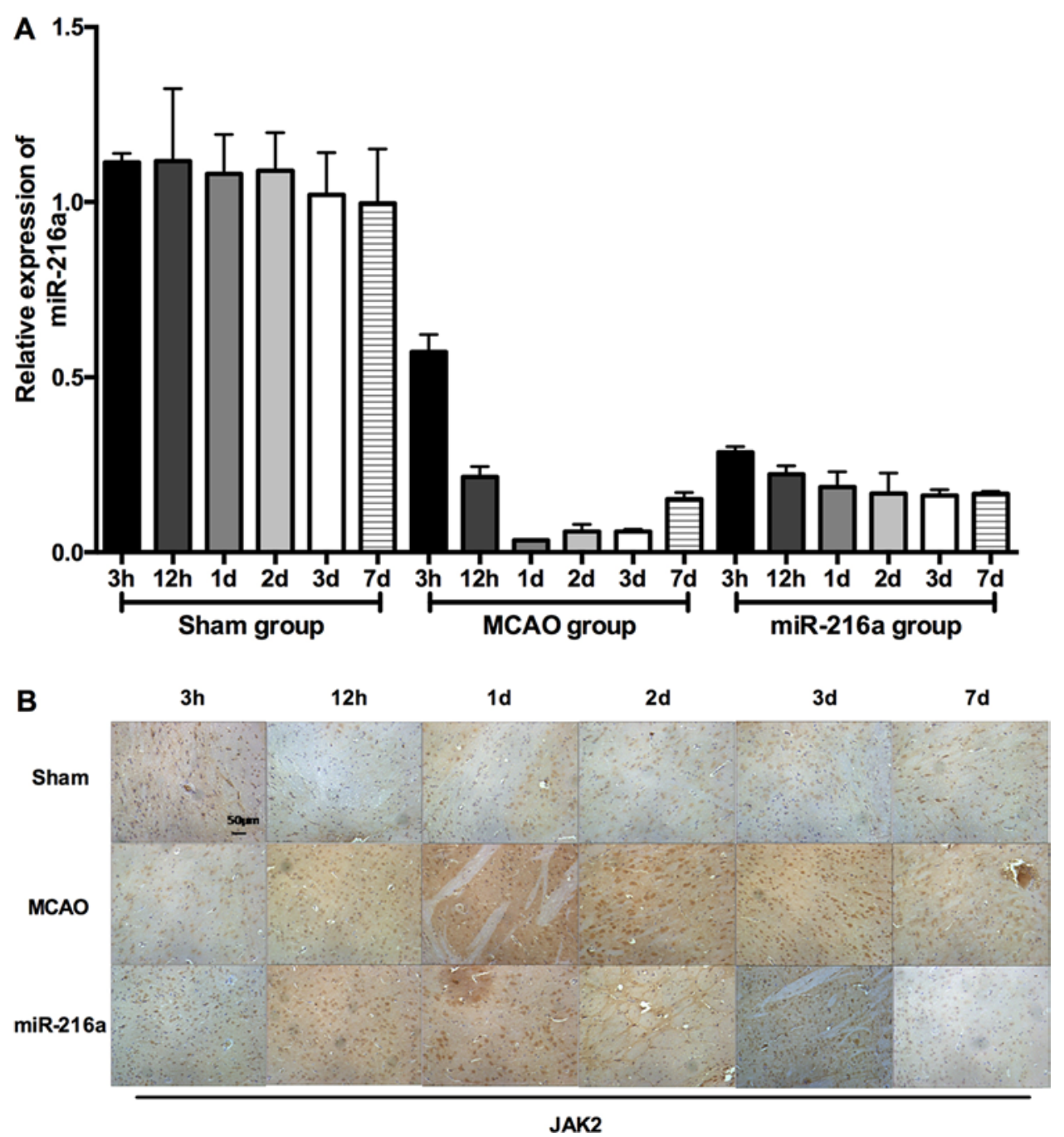

FIG. 5. Changes of JAK2 and miR-216a expression in the sham group, MCAO group, and miR-216a treatment group with 1 hour of ischemia following 24 hours of reperfusion at 3 hours, 12 hours, 1 day, 2 days, 3 days, and 7 days. A: The changes in miR-216a expression levels were measured using real-time qRT-PCR. U6 was used as an internal control for quantification; the expression of JAK2 protein was measured with immunohistochemistry. B: Representative immunohistochemical images of ischemia following 24 hours of reperfusion at 3 hours, 12 hours, 1 day, 2 days, 3 days, and 7 days. Figure is available in color online only.

as a tumor suppressor regulating pancreatic cancer cells by targeting the JAK/STAT pathway. ${ }^{9}$ Phosphorylation of the signal transducer and activator of STAT3 can also be regulated by miR-216a in pancreatic cancer, and miR-216a overexpression inhibited the JAK2/STAT3 signaling pathway and tumor growth in vivo. ${ }^{30}$ However, the inhibitory effect of miR-216a via the JAK2/STAT3 pathway in cerebral ischemia remains unclear.

In this study, we chose MTT, the TUNEL assay, and Western blotting to evaluate the effect of miR-216a on cell apoptosis. We also identified cleaved caspase-3 protein as a major indicator for cell apoptosis. The results showed that miR-216a can decrease the TUNEL-positive cells and level of cleaved caspase- 3 in vitro and in vivo, which was consistent with the results of knockdown of JAK2. Furthermore, our results showed that the ischemic infarction was obviously decreased in the stroke model of MCAO mice injected with miR-216a mimics and upregulation of miR-216a effectively improved neurologi- cal outcomes of mice. These results indicated that miR216a can contribute to the neuroprotective effect partly through targeting JAK2 in cerebral ischemia, which may provide a novel therapeutic approach for the treatment of ischemic injury.

Activation of the immune system was reported to play an important role in the pathophysiology of stroke, and severe ischemia can trigger an inflammatory cascade, leading to the infiltration of peripheral immune cells into brain tissue, aggravating postischemic inflammation and apoptosis. ${ }^{17,21,23}$ Using the OGD/R primary cultured neuronal model, we (for the first time) identified that overexpression of miR-216a can decrease JAK2/STAT3 downstream inflammatory mediators, including the inflammatory enzymes (iNOS and MMP-9) and cytokines (TNF- $\alpha$ and IL-1 $\beta$ ), and this effect can be restored by overexpression with JAK2. However, the exact relationship between miR216a and inflammatory mediators in patients with cerebral infarction needs to be investigated further. Because the ef- 
A

miR-216a

$3 \mathrm{~h}$

12

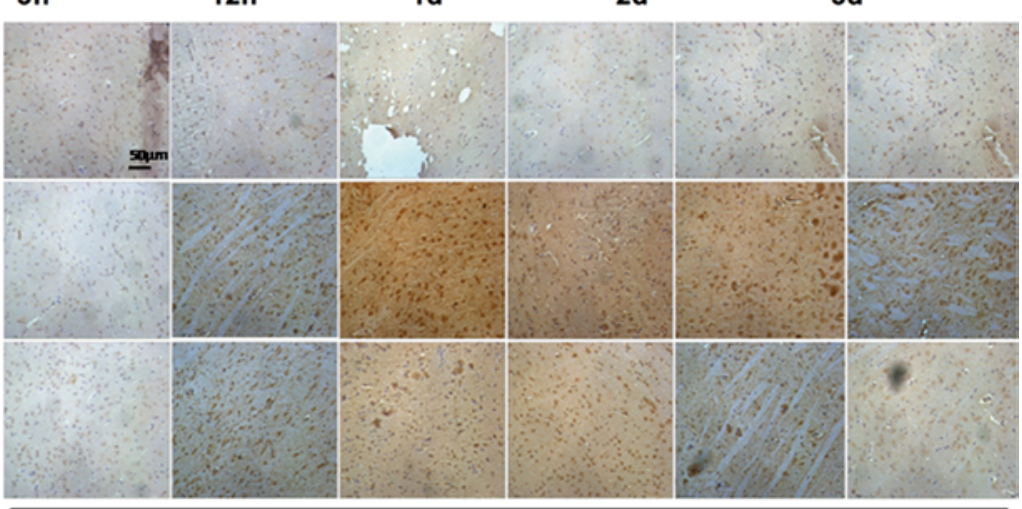

pSTAT3

B

Sham

MCAO

miR-216a

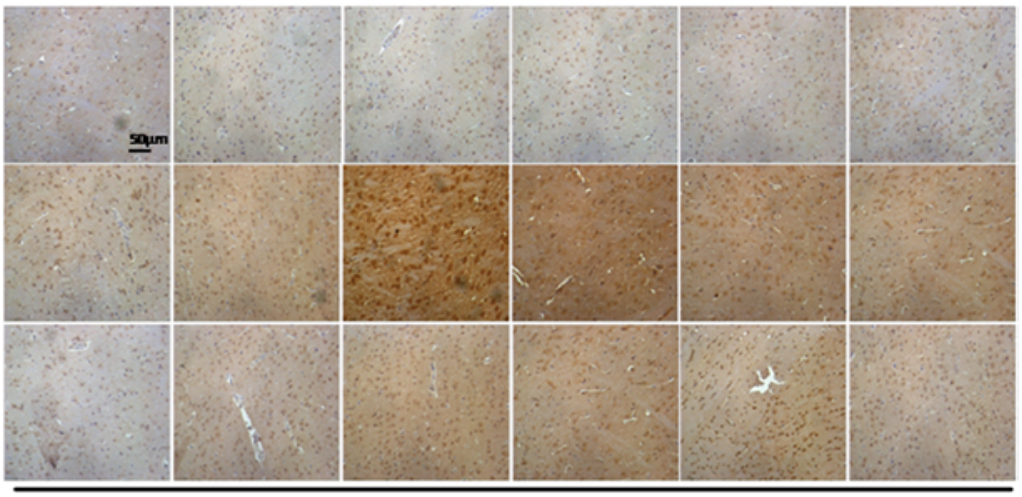

Cle Caspase 3

C

Sham

MCAO

miR-216a

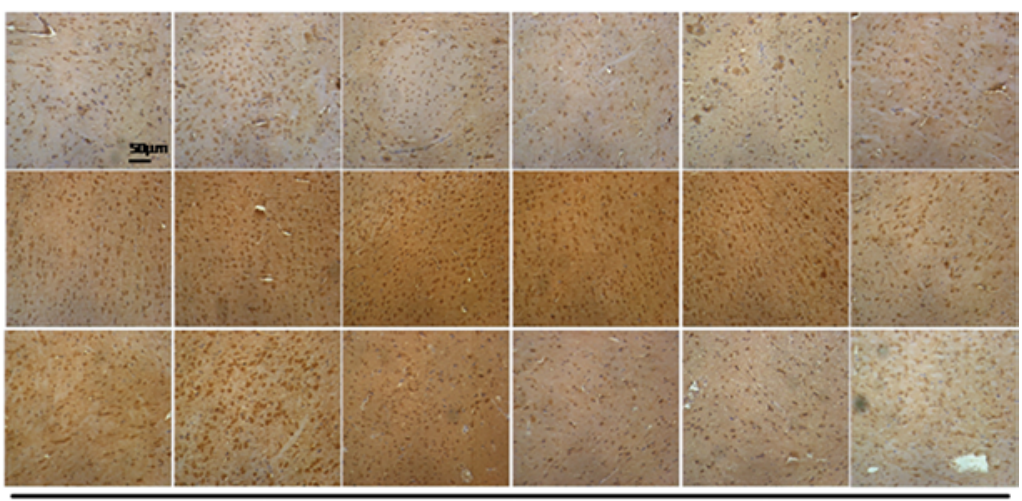

iNOS

FIG. 6. p-STAT3, cleaved caspase-3, and iNOS levels were detected by immunohistochemistry in sham, MCAO, and miR-216a treatment groups with 1 hour of ischemia following 24 hours of reperfusion. Compared with the sham group, the protein levels of p-STAT3 (A), cleaved caspase-3 (B), and NOS (C) of the MCAO group were increased and reached their highest levels on day 1, while miR-216a treatment reduced the protein levels. Figure is available in color online only.

fect of miR-216a may provide a novel therapeutic approach for the treatment of ischemic injury, further studies may pave the way for the clinical usage of miR-216a.

\section{Conclusions}

The findings of the present study indicated that miR216a exerts neuroprotective effects against ischemic injury in vivo and in vitro by negatively regulating JAK2/STAT3 downstream apoptosis and inflammatory pathways. How- ever, the exact molecular pathway remains to be studied in the future to provide new therapeutic opportunities for the treatment of ischemic stroke.

\section{Acknowledgments}

This work was supported by the National Natural Science Foundation of China (grant no. 30971015) and the Science and Technology Commission Foundation of Heilong Jiang Province (grant no. GC10C304-4). 

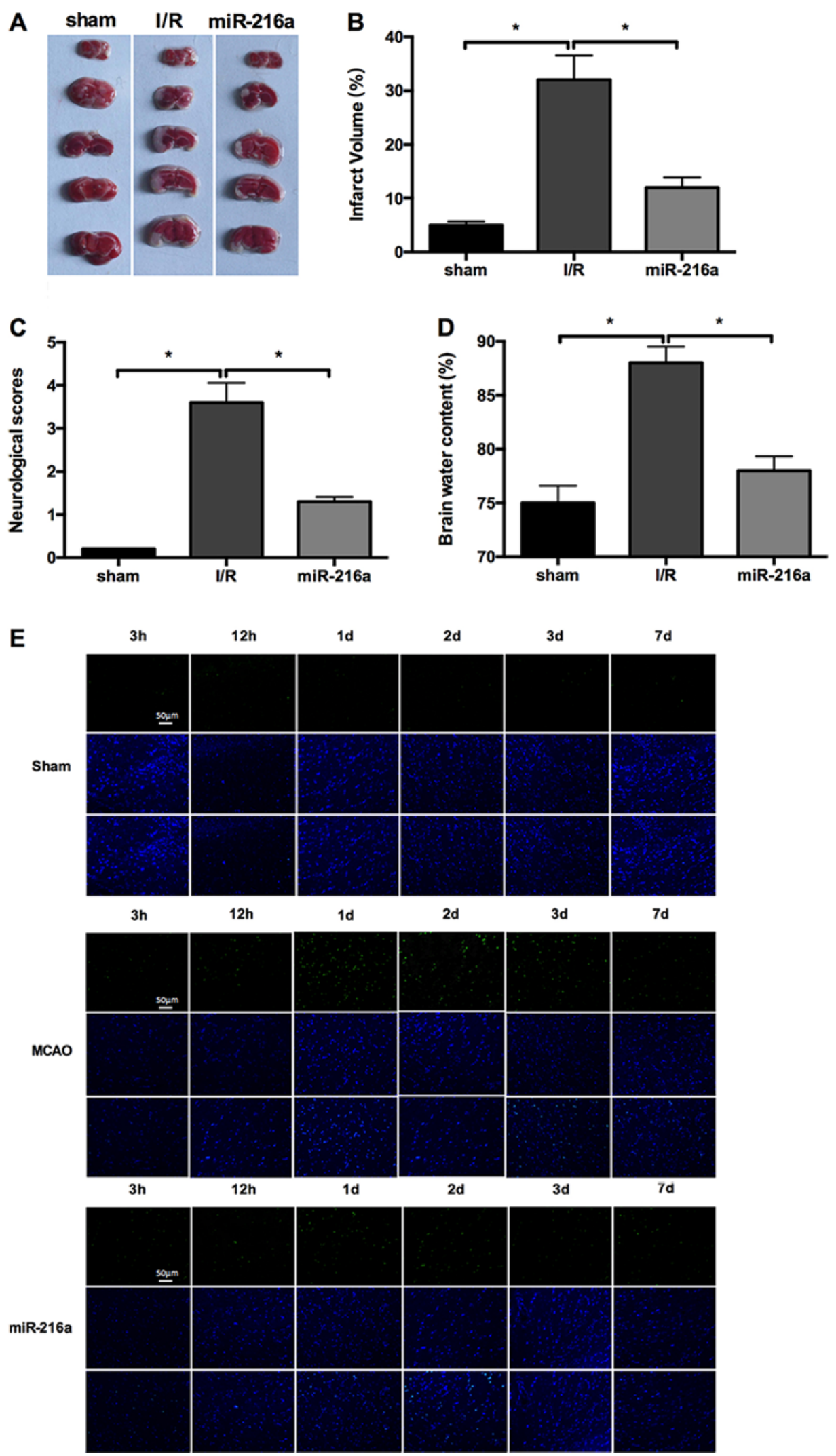

FIG. 7. miR-216a mimics attenuated infarct volumes, neurological dysfunction, and neuronal death. A: Ischemic injury was stained with TTC. B-D: Comparisons among the 3 groups for infarct volumes (B), neurological scores (C), and brain water content (D). E: Brain cell apoptosis was detected by TUNEL assay. $I / R=$ ischemia/reperfusion. ${ }^{*} p<0.05$. Figure is available in color online only. 


\section{References}

1. Baczyńska D, Michałowska D, Witkiewicz W: [The role of microRNA in ischemic diseases--impact on the regulation of inflammatory, apoptosis and angiogenesis processes.] Przegl Lek 70:135-142, 2013 (Polish)

2. Bartel DP: MicroRNAs: genomics, biogenesis, mechanism, and function. Cell 116:281-297, 2004

3. Buller B, Liu X, Wang X, Zhang RL, Zhang L, Hozeska-Solgot A, et al: MicroRNA-21 protects neurons from ischemic death. FEBS J 277:4299-4307, 2010

4. Chen YC, Wu JS, Yang ST, Huang CY, Chang C, Sun GY, et al: Stroke, angiogenesis and phytochemicals. Front Biosci (Schol Ed) 4:599-610, 2012

5. Dharap A, Bowen K, Place R, Li LC, Vemuganti R: Transient focal ischemia induces extensive temporal changes in rat cerebral microRNAome. J Cereb Blood Flow Metab 29:675-687, 2009

6. Dirnagl U, Iadecola C, Moskowitz MA: Pathobiology of ischaemic stroke: an integrated view. Trends Neurosci 22:391-397, 1999

7. Dong S, Yin H, Dong C, Sun K, Lv P, Meng W, et al: Predictive value of plasma microRNA-216a/b in the diagnosis of esophageal squamous cell carcinoma. Dis Markers 2016:1857067, 2016

8. Feigin VL, Norrving B, and Mensah GA: Global burden of stroke. Circ Res 120:439-448, 2017

9. Hou BH, Jian ZX, Cui P, Li SJ, Tian RQ, Ou JR: miR-216a may inhibit pancreatic tumor growth by targeting JAK2. FEBS Lett 589:2224-2232, 2015

10. Hou ST, MacManus JP: Molecular mechanisms of cerebral ischemia-induced neuronal death. Int Rev Cytol 221:93-148, 2002

11. Iadecola C, Alexander M: Cerebral ischemia and inflammation. Curr Opin Neurol 14:89-94, 2001

12. Jeyaseelan K, Lim KY, Armugam A: MicroRNA expression in the blood and brain of rats subjected to transient focal ischemia by middle cerebral artery occlusion. Stroke 39:959966, 2008

13. Li L, Li H, Li M: Curcumin protects against cerebral ischemia-reperfusion injury by activating JAK2/STAT3 signaling pathway in rats. Int J Clin Exp Med 8:14985-14991, 2015

14. Liang Z, Wu G, Fan C, Xu J, Jiang S, Yan X, et al: The emerging role of signal transducer and activator of transcription 3 in cerebral ischemic and hemorrhagic stroke. Prog Neurobiol 137:1-16, 2016

15. Lipton P: Ischemic cell death in brain neurons. Physiol Rev 79:1431-1568, 1999

16. Liu X, Li F, Zhao S, Luo Y, Kang J, Zhao H, et al: MicroRNA-124-mediated regulation of inhibitory member of apoptosis-stimulating protein of p53 family in experimental stroke. Stroke 44:1973-1980, 2013

17. Macrez R, Ali C, Toutirais O, Le Mauff B, Defer G, Dirnagl $\mathrm{U}$, et al: Stroke and the immune system: from pathophysiology to new therapeutic strategies. Lancet Neurol 10:471-480, 2011

18. Palmer GC, Peeling J, Corbett D, Del Bigio MR, Hudzik TJ: T2-weighted MRI correlates with long-term histopathology, neurology scores, and skilled motor behavior in a rat stroke model. Ann N Y Acad Sci 939:283-296, 2001

19. Peng Z, Li J, Li Y, Yang X, Feng S, Han S, et al: Downregulation of miR-181b in mouse brain following ischemic stroke induces neuroprotection against ischemic injury through tar- geting heat shock protein A5 and ubiquitin carboxyl-terminal hydrolase isozyme L1. J Neurosci Res 91:1349-1362, 2013

20. Reglodi D, Tamás A, Lengvári I: Examination of sensorimotor performance following middle cerebral artery occlusion in rats. Brain Res Bull 59:459-466, 2003

21. Sairanen T, Karjalainen-Lindsberg ML, Paetau A, Ijäs P, Lindsberg PJ: Apoptosis dominant in the periinfarct area of human ischaemic stroke-a possible target of antiapoptotic treatments. Brain 129:189-199, 2006

22. Schmittgen TD, Livak KJ: Analyzing real-time PCR data by the comparative C(T) method. Nat Protoc 3:1101-1108, 2008

23. Shichita T, Sakaguchi R, Suzuki M, Yoshimura A: Post-ischemic inflammation in the brain. Front Immunol 3:132, 2012

24. Stoll G, Jander S, Schroeter M: Detrimental and beneficial effects of injury-induced inflammation and cytokine expression in the nervous system. Adv Exp Med Biol 513:87-113, 2002

25. Sun SL, Li TJ, Yang PY, Qiu Y, Rui YC: Modulation of signal transducers and activators of transcription (STAT) factor pathways during focal cerebral ischaemia: a gene expression array study in rat hippocampus after middle cerebral artery occlusion. Clin Exp Pharmacol Physiol 34:1097-1101, 2007

26. Wang L, Zhuang L, Rong H, Guo Y, Ling X, Wang R, et al: MicroRNA-101 inhibits proliferation of pulmonary microvascular endothelial cells in a rat model of hepatopulmonary syndrome by targeting the JAK2/STAT3 signaling pathway. Mol Med Rep 12:8261-8267, 2015

27. Wang S, Chen X, Tang M: MicroRNA-216a inhibits pancreatic cancer by directly targeting Janus kinase 2 . Oncol Rep 32:2824-2830, 2014

28. Wang Y, Ren X, Deng C, Yang L, Yan E, Guo T, et al: Mechanism of the inhibition of the STAT3 signaling pathway by EGCG. Oncol Rep 30:2691-2696, 2013

29. Xia H, Ooi LL, Hui KM: MicroRNA-216a/217-induced epithelial-mesenchymal transition targets PTEN and SMAD7 to promote drug resistance and recurrence of liver cancer. Hepatology 58:629-641, 2013

30. Yin KJ, Deng Z, Huang H, Hamblin M, Xie C, Zhang J, et al: miR-497 regulates neuronal death in mouse brain after transient focal cerebral ischemia. Neurobiol Dis 38:17-26, 2010

31. Zhang X, Shi H, Lin S, Ba M, Cui S: MicroRNA-216a enhances the radiosensitivity of pancreatic cancer cells by inhibiting beclin-1-mediated autophagy. Oncol Rep 34:15571564,2015

\section{Disclosures}

The authors report no conflict of interest concerning the materials or methods used in this study or the findings specified in this paper.

\section{Author Contributions}

Conception and design: Liu, Jin. Acquisition of data: Sun. Analysis and interpretation of data: Zhong, Wang. Drafting the article: Tian. Approved the final version of the manuscript on behalf of all authors: Li. Statistical analysis: Zhao.

\section{Correspondence}

Guo Zhong Li: Harbin Medical University, Harbin, Heilong Jiang Province, China.1gzhyd1962@163.com. 